\title{
A high proportion of prehospital emergency patients are not transported by ambulance: a retrospective cohort study in Northern Finland
}

\author{
Marko Hoikka $^{1}$, Tom Silfvast ${ }^{2}$, Tero I Ala-Kokko ${ }^{3}$
}

${ }^{1}$ Medical Research Center, Research Group of Surgery, Anesthesia and Intensive Care, University of Oulu and Department of Anesthesiology, Division of Intensive Care, Oulu University Hospital, Finland. PO BOX 21, FI-90029 OYS. E-mail: marko.hoikka@ student.oulu.fi.

${ }^{2}$ University of Helsinki and Emergency Medical Service, Department of Emergency Medicine, Helsinki University Central Hospital, Finland. FI-00029 HUS. E-mail: tom.silfvast@ hus.fi.

${ }^{3}$ Medical Research Center, Research Group of Surgery, Anesthesia and Intensive Care, University of Oulu and Department of Anesthesiology, Division of Intensive Care, Oulu University Hospital, Finland. PO BOX 21, FI-90029 OYS. E-mail: tero.ala-kokko@ppshp.fi.

Correspondence with:

Marko Hoikka, MD, PhD-student

Department of Anesthesiology, Division of Intensive Care, Oulu University Hospital PO BOX 21, 90029 OUH, Finland

E-mail: marko.hoikka@student.oulu.fi

All authors designed the study, analyzed and interpreted the data, drafted and critically revised the manuscript. MH collected and extracted the data. All authors have read and approved the final version of the manuscript.

All authors declare that they have no financial or any other conflicts of interests.

Acknowledgements: The EMS personnel who collected the data while taking care of the patients are highly acknowledged. The statistical help of Mr. Pasi Ohtonen, MSc, during the design and analysis of the study is appreciated. This study was funded by an EVO grant from Oulu University.

Short title: Non-transportation in EMS

Word count: 2460 


\begin{abstract}
Background: The number of missions in the emergency medical services (EMS) has increased considerably in the recent years. People are requesting ambulance for even minor illnesses and nonmedical problems, which is placing financial and resource burdens on the EMS. The aim of this study was to determine the rate of non-transportation missions in Northern Finland and the reasons for these missions.
\end{abstract}

Methods: All ambulance missions in two hospital districts in Northern Finland during the six-month period of January 1 through June 30, 2014, were retrospectively evaluated from the EMS charts to identify missions in which the patients were not transported by the EMS. The non-transportation rates and reasons were calculated and expressed as percentages.

Results: In $41.7 \%$ of the 13354 missions the patient was not transported from the scene by an ambulance. After a medical assessment and care was provided by the EMS, $48.2 \%$ of these nontransport patients were evaluated as not needing further treatment in the emergency department and were directed to contact the municipal health care center during office hours. There was no need for any medical care in $39.9 \%$ of non-transportation missions.

Conclusion: This study showed a high rate of EMS missions resulting in non-transportation in two hospital districts in Northern Finland. In the majority of these missions there was no need for emergency admission to an emergency department or for any medical care at all. These findings indicate that an improvement in the dispatch process and primary care resources might be of benefit.

Key words: Emergency Medical Services, Ambulance Diversion, Emergency Medical Service Communication Systems 


\section{Introduction}

The number of missions in the emergency medical services (EMS) has increased considerably in recent years. ${ }^{1,2}$ People increasingly contact emergency medical communication centers (EMCCs) and request an ambulance for even minor illnesses or injuries, as shown by a recent review. ${ }^{3}$ Adequate risk assessment and emergency dispatching play key roles in EMS utilization, EMS resource management and costs. ${ }^{4}$ Triage at dispatch has to be sufficiently sensitive to identify true lifethreatening situations, but over-triaging and merely sending ambulances on-request may lead to an inappropriate use of available EMS resources.

In recent decades the rate and nature of non-transportation EMS missions have changed. Several EMS providers in the UK did not previously allow EMS crews to leave patients non-transported, unless the patient refused transportation. ${ }^{5}$ Such a policy may lead to overcrowding of the emergency departments; for example, in the US $70 \%$ of transported patients are discharged home from the emergency departments. ${ }^{6}$ Because the health care system is imposing a widened range of tasks and interventions to be performed by the EMS, the scope of EMS practice has changed, and guidelines for non-transportation situations have been implemented. ${ }^{5,7}$ In Finland, guidelines enable EMS providers to make liberal non-transportation decisions based on the patient's actual needs, which may lead to a high proportion of missions in which transportation by ambulance is not indicated.

In Finland municipal health care centers are responsible for urgent outpatient medical care during office hours (Mon-Fri 8:00 AM - 4:00 PM), whereas care outside office hours is centralized to the regional hospitals. The hospital network is currently undergoing a reform, which creates larger but fewer units for the provision of emergency care overall. For many patients, this means longer distances to the closest outpatient clinic or hospital. In Northern Finland this will result in distances longer than $100 \mathrm{~km}$ for some patients, which may affect the use of EMS services.

The main objective of this study was to determine the rate and causes of non-transportation missions in two Finnish emergency medical systems. Secondarily, we wanted to explore whether there is a relationship between the availability of and the distance to health care services and the use of EMS. 


\section{Methods}

This retrospective registry study was carried out in two similar hospital districts and EMS-systems (Kainuu and Länsi-Pohja) in Northern Finland (Figure 1).

In Finland, there is a national dispatch authority with six regional EMCCs. The common European emergency number 112 is used for all emergencies requiring urgent assistance, whether from police, rescue, medical care or social services. EMCC personnel receive a national 18-month formalized training without an official status as health care professionals. The same EMCC dispatcher works as a call handler as well as a dispatcher for all authorities throughout the emergency call. All incoming calls for medical emergencies are assessed according to a criteria-based, nationally standardized dispatch protocol. The EMS calls are prioritized in four categories, A, B, C, and D, where A indicates an evident or suspected life-threatening situation, B other high-risk situation, $\mathrm{C}$ other urgent situation, and $\mathrm{D}$ a non-urgent situation.

The study areas consist mostly of suburban and rural populations. A total of 140000 inhabitants, representing $2.6 \%$ of the Finnish population, live in these areas with a population density of 4.7 inhabitants per square kilometer. There are 6 to 8 municipal health care centers and one regional hospital in both districts. Both districts are covered by a single EMCC (Oulu) which dispatches a total of 35000 EMS missions in the study areas annually. The districts share similar 3-tier EMS systems, organized by the hospital districts. The EMS providers have written guidelines for documenting the reasons for non-transportation situations. The decisions to not transport the patient are made after examining the patient and excluding disturbance of vital functions, and after consultation with an oncall emergency department physician according to protocol.

The data for this study were collected retrospectively from all EMS charts in the two study districts between January 1 and June 30, 2014. In the Kainuu region, the main author manually transferred the data from the paper EMS charts to the statistical program, whereas in the Länsi-Pohja region the data were electronically transferred from the EMS database (Merlot Medi®, CGI, Canada). All dispatched EMS missions within every priority category were included, whereas secondary (inter-facility) transports and missions of home care assistance were excluded. The EMS missions that were cancelled before patient contact were also excluded. Collected data included priority and dispatch code, demographic data and the non-transportation code. Patients with multiple non-transport missions were also identified. Distances to hospital were calculated using the municipal population 
centroid, not the patient's actual home address. General information on health care use in the regions was obtained from the National Institute for Health and Welfare, which produces a range of statistics in the fields of social welfare and health care to support decision making, development and research. As a statistical authority it is responsible for the maintenance and development of statistical and register resources.

The study design was observational and no clinical interventions were performed. Therefore, according to the local policies, approval of the local ethics committee was waived. Permission to carry out the study was obtained from the Hospital Districts (20 Mar 2014; 8 Apr 2014) and the Office of Data Protection Ombudsman_(dnro 719/4225/2014).

\section{Statistical analyses}

Statistical analyses were performed with SPSS Statistics, version 22 (IBM Corp., Armonk, NY). Data were expressed as mean with standard deviation, unless otherwise stated. Categorical variables were expressed as percentages, and Fisher's exact test was used for statistical comparison. A two-tailed $P$ value of less than 0.05 was considered statistically significant. The Spearman's correlation coefficient was used to evaluate the association between distance to the emergency department and rate of nontransportation missions. 


\section{Results}

A total of 13354 EMS missions fulfilled the inclusion criteria during the six-month study period (Figure 2). Three-quarters (10 332) of the missions were due to illness or disease, while the remaining missions were related to various traumas. Of the patients, $50.7 \%$ were male, and the median age of all patients was 68 years.

The rates and the reasons for non-transportation missions are presented in Table 1 . There were 5570 missions (41.7\%) that did not lead to transportation by ambulance. In almost half of them (48.2\%), the patient was evaluated by the EMS to not require acute treatment in the emergency department and was instructed to contact the municipal health care center during office hours. In another 1891 patients (33.9\% of the non-transported patients), there was no need for medical care at all, and 509 patients (9.1\% of the non-transported patients) were considered to need assessment or treatment in the emergency department but were directed to use vehicles other than ambulance (e.g., taxi, a relative's car).

During office hours (Mon-Fri 8:00 AM - 4:00 PM) the non-transportation rate (NTR) was 29.3\% (95\%CI 27.9\%-30.7\%) and outside office hours it was $46.9 \%(95 \%$ CI $45.9 \%-48.0 \%)(\mathrm{p}<0.001)$. Figure 3 shows the NTR in relation to the time of the EMS mission. The NTR outside office hours increased with longer distances to the emergency department (Spearman $\rho=0.656, p=0.008$ ) (Figure 4). The NTR in the Kainuu region was $46.2 \%$ (95\%CI 45.1\%-47.3\%) and that in the Länsi-Pohja region was $32.3 \%(95 \%$ CI 30.9\%-33.6\%) (p<0.001).

During the six-month study period, $28.8 \%$ of the non-transportation patients had multiple contacts with EMS resulting in non-transportation missions. The highest count of non-transportation missions for one patient was 45 . 


\section{Discussion}

This study showed that four out of ten EMS missions did not lead to ambulance transportation of the patient. In almost half of these cases the patients could stay at home after the medical assessment by the EMS crew and contact their municipal health care center later with an non-urgent matter, while one-third of the non-transportation patients did not need any medical care at all. The NTR was higher outside office hours and it increased with the distance to the emergency department. These findings necessitate a discussion as to the dispatch process and the reasons why people with minor illnesses request an ambulance.

The limitations of this study relate to the fact that the study was retrospective, and therefore the registries used were not specifically designed to explore those issues under examination. The available data, however, which were initially documented for administrative and medico-legal purposes, were of good quality and had no data missing. Human factors in the EMS system may distort the results, as the compliance of EMCC dispatchers and EMS providers with the predefined dispatching protocols and non-transportation guidelines could not be documented during the study. The strengths of this study pertain to the large cohort from two different hospital districts.

The finding that more than $80 \%$ of the non-transported patients could be left at the scene and instructed to contact their primary health care center during office hours, or did not need any health care at all, highlights the difficulty in assessing a patient's condition over the phone. It may be difficult to obtain adequate information from the caller, but one must also ask whether the current dispatch protocols, the dispatchers' education and dispatch centers' resources are optimal. The dispatch protocols aim at identifying acute illness or injury, and in most non-transported patients the nature of the problem (chronic disease or a social issue) was less urgent. The protocol used may not be sufficiently accurate to identify such problems. In patients with high-risk symptoms (e.g., chest pain, shortness of breath), however, it is reasonable to dispatch EMS to evaluate the patient's condition at the scene, even if in retrospect the mission did not require transportation of the patient.

On the other hand, the dispatcher's education plays a significant role. The current national 18-month dispatcher training in Finland may need to be refocused on the changing needs in the evaluation of requests for EMS, as the advisory and assessment tasks in less urgent situations appears to become increasingly in demand. As the current Finnish EMCC dispatchers are not health care professionals and therefore not covered by the legal framework the same way as health care professionals are, they 
may experience a fear of prosecution, and this in turn may lower the threshold for dispatching an ambulance. There are differences between the Nordic countries with regard to the organization of emergency medical dispatch systems as well as the use of dispatch protocols. ${ }^{8}$ The level of education of the EMCC personnel varies from a few weeks' training to that of health care professionals — nurses or paramedics - who handle medical emergency calls. A study by Forslund et al showed that when health care professionals provided informational support to the dispatchers who were dealing with non-urgent calls to the dispatch center, the number of missions leading to non-transportation was reduced. ${ }^{9}$

It is unclear why people with minor illnesses or non-medical problems request an ambulance. This inappropriate utilization of ambulance services has been a worldwide problem, ${ }^{1}$ and the phenomenon has been known since the 1970s. ${ }^{10}$ In our series there was no need for medical care or interventions in one-third of the non-transportation missions. These missions represented $14.2 \%$ of all EMS missions. In addition, $3.8 \%$ of all patients were directed to use vehicles other than an ambulance. In a Swedish study, ambulance personnel estimated that the usage rate of taxis or other vehicles could be even higher, up to $31 \%$ of the missions. ${ }^{11}$ One reason for the high NTR could be that the primary health care is unable to meet the needs of the patient. During the study, there was a severe shortage of primary care physicians in the study districts (Kainuu 20.8\% and Länsi-Pohja $8.3 \%$ ), and 96\% of the population in Kainuu experienced difficulties in getting a doctor's appointment on a weekly basis, ${ }^{12}$ Moreover, several ambulance visits to the same patient without the need for transportation may indicate that the patient's problems have not been adequately dealt with in primary health and social care. An association between a lack of primary care resources and the regional unfocused use of EMS may be a sign to decision makers that primary care must be strengthened. The high NTR implies that the EMS compensates for the lack of primary care resources in some regions although this has not originally been the intention in the healthcare system.

Another reason for the high NTR might be the distances involved in these regions. We found a positive correlation between the distance to the emergency department and NTR (Figure 4). Especially outside office hours, when municipal health care centers are closed, people may prefer to call for an EMS unit rather than make their way to a distant emergency department. Similar findings were found in a Norwegian study in which increased distance was associated with lower rates of all contact types to casualty clinics except telephone consultations by a doctor. ${ }^{13}$ This correlation between NTR outside office hours and the distance to the emergency department was an interesting finding which may be difficult to interpret. Could it be that the threshold for transporting a patient a 
long distance is higher at night than during daytime? If so, can such a pattern be ascribed to the fact that an EMS unit is away from its response area for a lengthy time and the reluctance to transportation is therefore higher? Or is the threshold for dispatching an ambulance lower at night?

The results of this study indicate a need for further studies - most importantly, studies that look into patient safety issues in an EMS system with such a high NTR. Data as to whether these non-transport decisions are correct or affect patient outcome are lacking. Studies are also needed to evaluate the extent to which involvement of health care professionals in EMCCs reduces the number of missions that do not necessitate an EMS response. The feasibility, safety and cost-effectiveness of forwarding non-urgent requests and missions to telephone health advisors, home care, or paramedicine providers, for example, should be studied. The use of other than EMS professionals for the evaluation of patients on the scene in less urgent situations is becoming increasingly common in Finland, corresponding with reports from several programs on community paramedicine. ${ }^{14}$ Although the results cannot be generalized to other health care systems, our study indicates that there is a need for studies evaluating the overall provision of EMS in healthcare systems.

\section{Conclusion}

This study showed a high rate of non-transportation missions in two Finnish EMS systems. In half of these non-transportation missions the EMS personnel assessed that there was no need for emergency admission to an emergency department, while in another third there was no need for any medical care at all. These findings indicate that an improvement in the dispatch process and primary care resources might be of benefit, especially during out-of-office hours. 


\section{References}

1. Lowthian JA, Cameron PA, Stoelwinder JU, Curtis A, Currell A, Cooke MW, McNeil JJ. Increasing utilisation of emergency ambulances. Australian Health Review 2011; 35:63-9.

2. Pittet V, Burnand B, Yersin B, Carron P-. Trends of pre-hospital emergency medical services activity over 10 years: A population-based registry analysis. BMC Health Serv Res 2014; 14.

3. Booker MJ, Shaw ARG, Purdy S. Why do patients with 'primary care sensitive' problems access ambulance services? A systematic mapping review of the literature. BMJ Open 2015; 5.

4. Ornato JP. Science of emergency medical dispatch. Circulation 2009; 119:2023-5.

5. Snooks HA, Dale J, Hartley-Sharpe C, Halter M. On-scene alternatives for emergency ambulance crews attending patients who do not need to travel to the accident and emergency department: A review of the literature. Emerg Med J 2004; 21:212-5.

6. Hettinger AZ, Cushman JT, Shah MN, Noyes K. Emergency medical dispatch codes association with emergency department outcomes. Prehosp Emerg Care 2013; 17:29-37.

7. Gray JT, Wardrope J. Introduction of non-transport guidelines into an ambulance service: a retrospective review. Emergency Medicine Journal 2007; 24:727-9.

8. Langhelle A, Lossius HM, Silfvast T, Björnsson HM, Lippert FK, Ersson A, Søreide E. International EMS Systems: the Nordic countries. Resuscitation 2004; 61:9-21.

9. Forslund K, Kihlgren M, Sorlie V. Experiences of adding nurses to increase medical competence at an emergency medical dispatch centre. Accid Emerg Nurs 2006; 14:230-6.

10. Morris DL, Cross AB. Is the emergency ambulance service abused? Br Med J 1980; 281:121-3.

11. Hjälte L, Suserud B-, Herlitz J, Karlberg I. Why are people without medical needs transported by ambulance? A study of indications for pre-hospital care. Eur J Emerg Med 2007; 14:151-6.

12. The National Institute for Health and Welfare. Statistical database. $2015 ; 2015$.

13. Raknes G, Hansen EH, Hunskaar S. Distance and utilisation of out-of-hours services in a Norwegian urban/rural district: an ecological study. BMC Health Services Research 2013; 13:222.

14. Choi BY, Blumberg C, Williams K. Mobile Integrated Health Care and Community Paramedicine: An Emerging Emergency Medical Services Concept. Ann Emerg Med 2016; 67:361-6. 
Figure 1. Map of Northern Finland and the hospital districts.

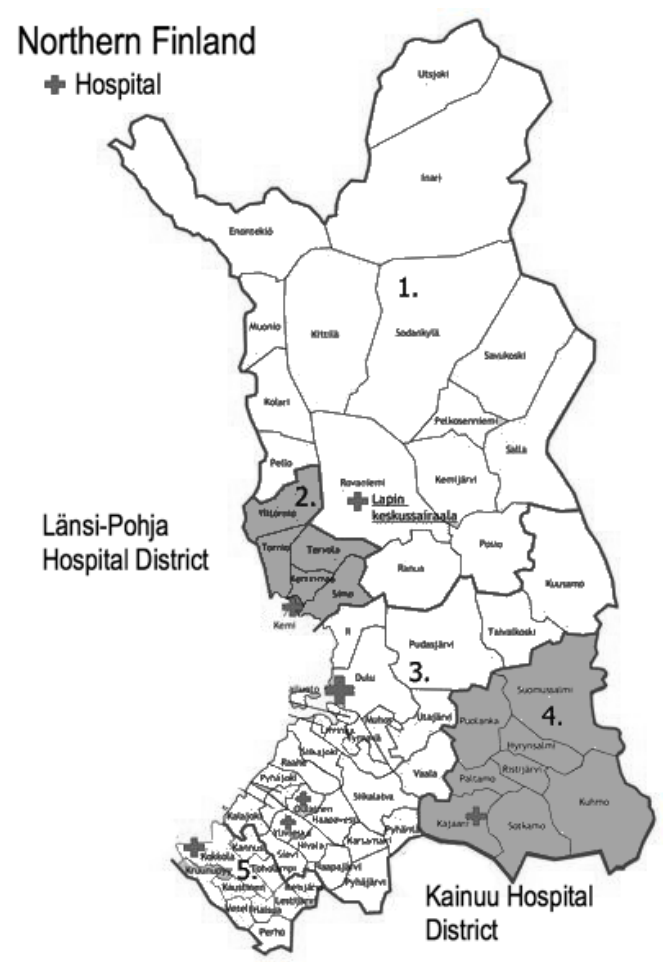


Figure 2. Flowchart of study selection and distribution of missions by priority categories and nontransportation rates. Priority A, evident or suspected life-threatening situation; priority B, other high-risk situation; priority $\mathrm{C}$, other urgent situation; priority $\mathrm{D}$, non-urgent situation.

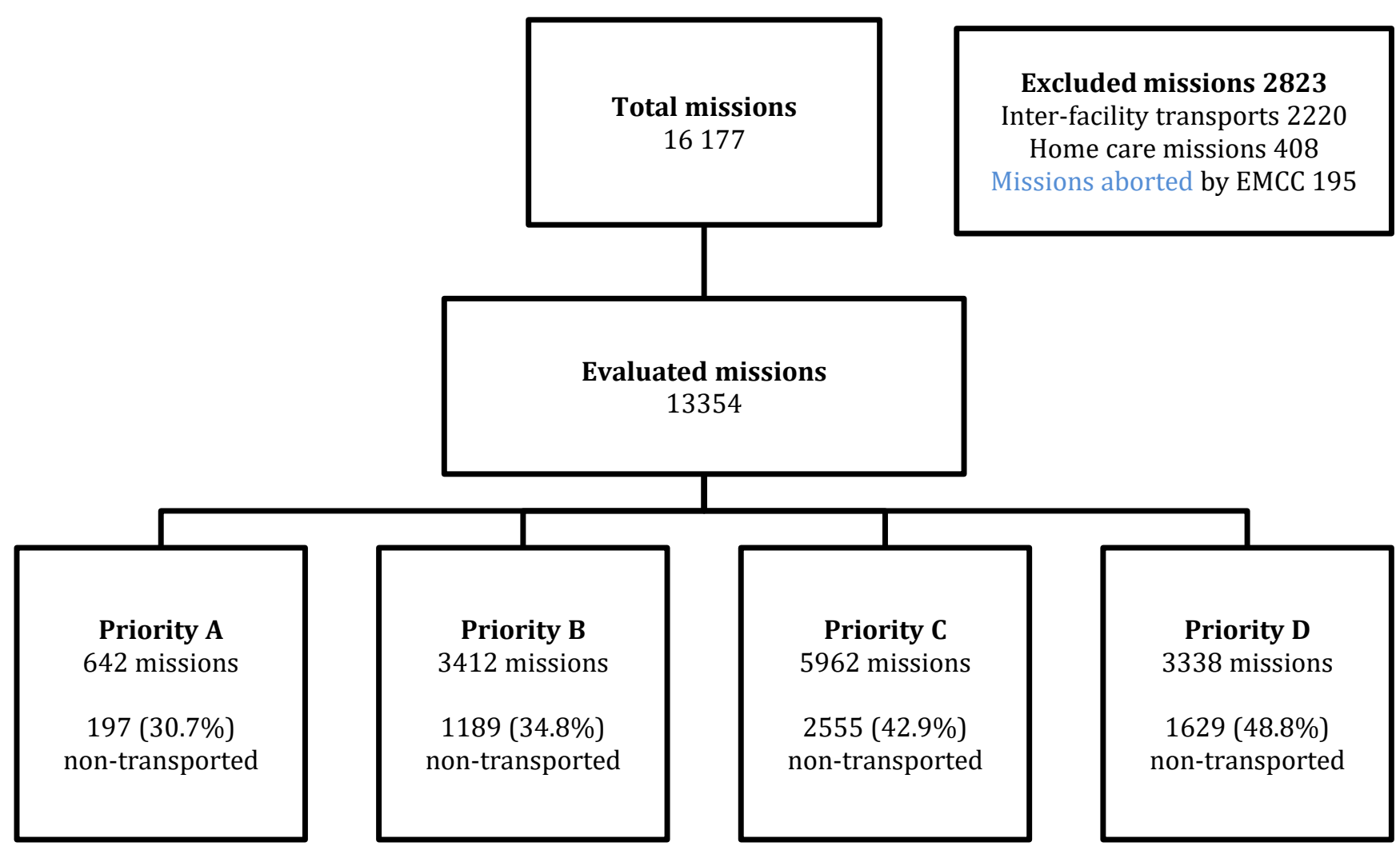


Figure 3. Non-transportation rate (bars) and number of missions (line) in relation to the time of the EMS mission.

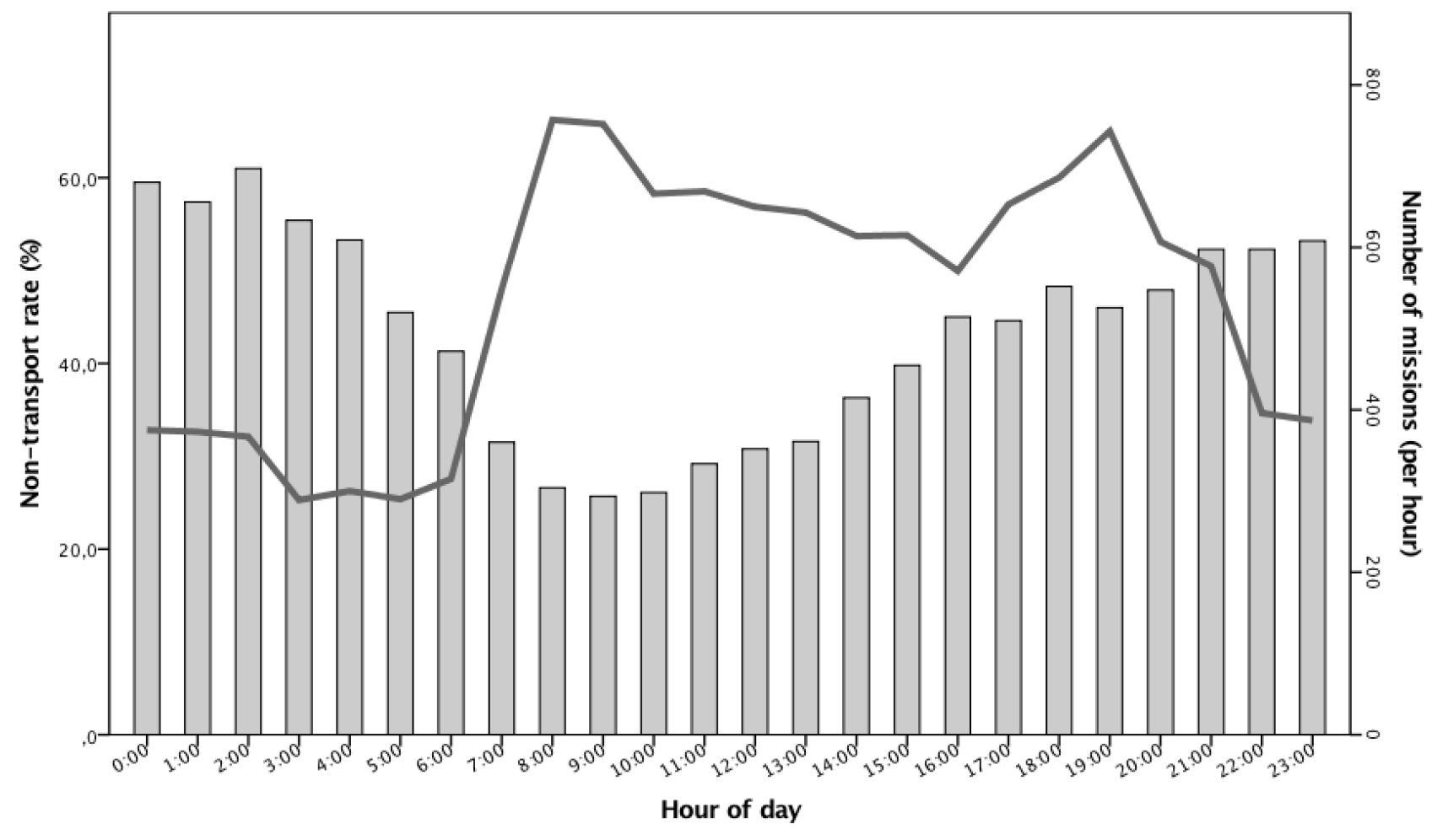


Figure 4. Non-transportation rate in relation to the distance between the municipal population centroid and the emergency department during office hours and outside office hours. Spearman's correlation coefficient $\rho=0.220, \rho=0.656(p=0.431, p=0.008)$.

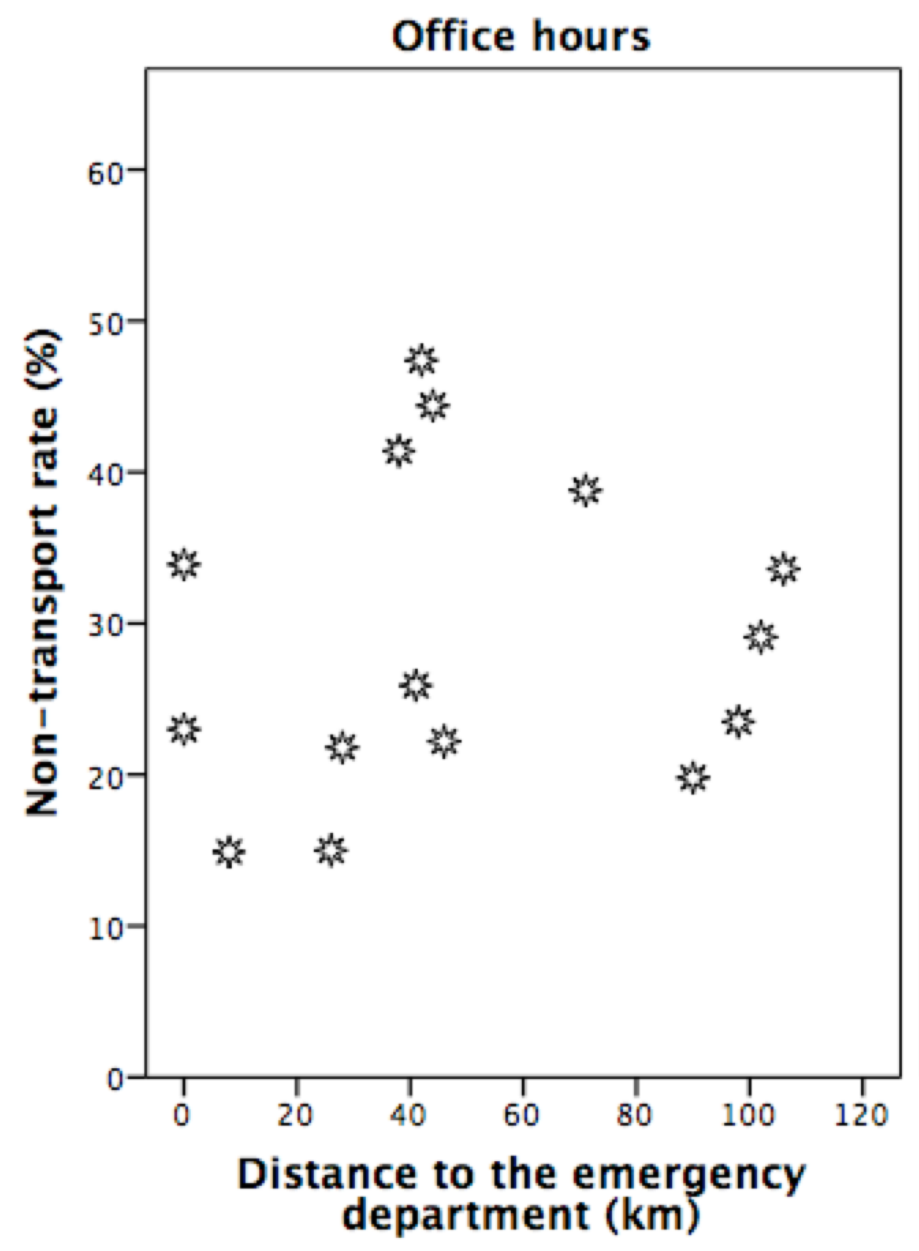

Outside office hours

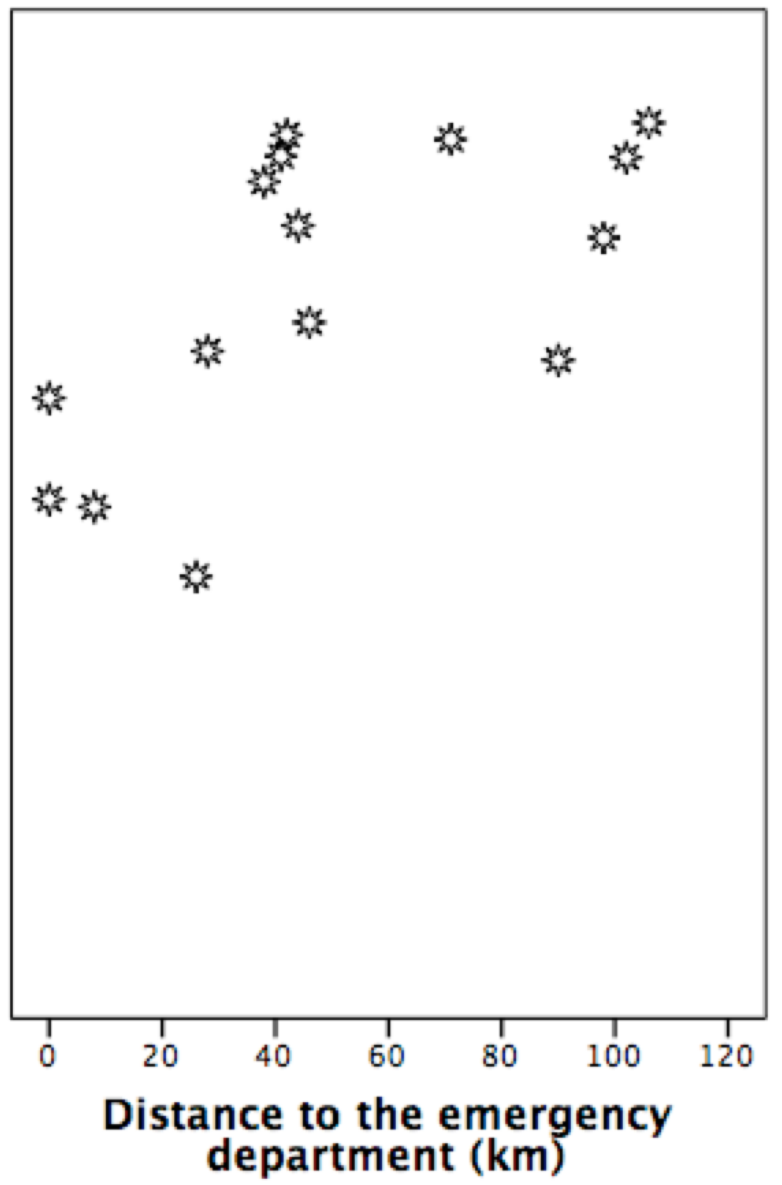

